\title{
Supplementary Material for \\ Virtual Homonuclear Decoupling in Direct Detection Nuclear Magnetic Resonance Experiments using Deep Neural Networks
}

Gogulan Karunanithy ${ }^{1, *}$, Harold W Mackenzie ${ }^{1}$ and D Flemming Hansen ${ }^{1, *}$

1) Institute of Structural and Molecular Biology, Division of Biosciences, University College London, London, UK WC1E 6BT

To whom correspondence should be addressed:

D. Flemming Hansen; $\quad$ E-mail: d.hansen@ucl.ac.uk

Gogulan Karunanithy; $\quad$ E-mail: g.karunanithy@ucl.ac.uk

\section{Contents}

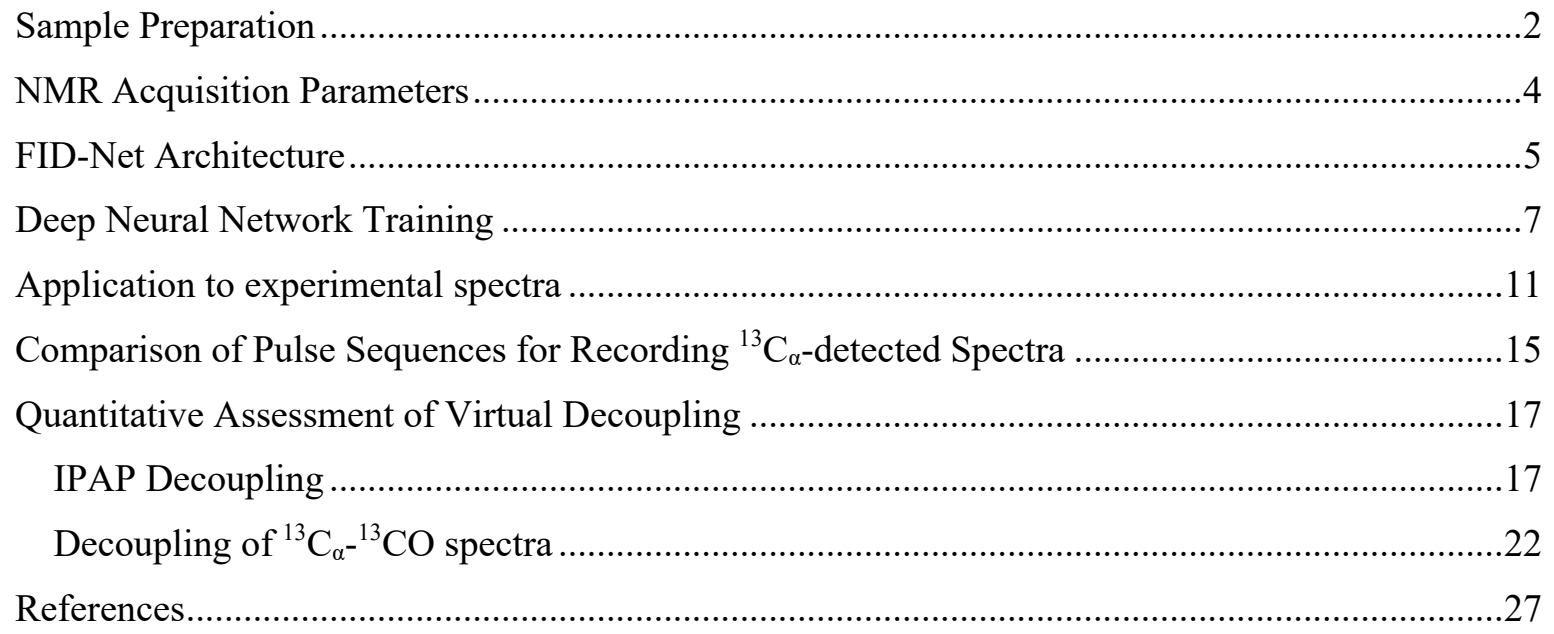




\section{Sample Preparation}

Isotopically labelled T4 lysozyme mutant C54T/C97A/L99A (T4L99A) was expressed and purified as described previously ${ }^{[1]}$. Briefly, a codon- optimised form of the gene containing the mutations L99A, C54A and C97A in a kanamycin-resistant, pET-29b vector was transformed into BL21 (DE3) chemically competent $E$. coli cells. A single colony was used to inoculate a $5 \mathrm{~mL}$ culture, which was grown overnight at $37{ }^{\circ} \mathrm{C}$. This was used to inoculate $50 \mathrm{~mL}$ of minimal M9 media made with ${ }^{2} \mathrm{H}_{2} \mathrm{O}$ and supplemented with $1 \mathrm{~g} \mathrm{~L} \mathrm{~L}^{-1}\left[{ }^{1} \mathrm{H},{ }^{15} \mathrm{~N}\right]$-ammonium chloride as the sole nitrogen source and $2 \mathrm{~g} \mathrm{~L}^{-1}\left[{ }^{2} \mathrm{H},{ }^{13} \mathrm{C}_{6}\right]$-glucose as the sole carbon source. This culture was grown overnight at $37^{\circ} \mathrm{C}$ and used to inoculate a final $2 \mathrm{~L}$ culture. The final culture was grown to $\mathrm{OD}_{600} \approx 0.6$ before a $16 \mathrm{~h}$ induction with $1 \mathrm{mM} \mathrm{IPTG}$ at $18{ }^{\circ} \mathrm{C}$. The T4L L99A protein was initially purified by ion-exchange chromatography. After lysis by sonication (in $50 \mathrm{mM} \mathrm{NaPO}_{4}$ (pH 6.5), $2 \mathrm{mM}$ EDTA, $5 \mathrm{mg}$ of DNAse1 (Sigma) and 1 cOmplete ${ }^{\mathrm{TM}}$ Mini Protease Inhibitor Cocktail tablet (Sigma) per $50 \mathrm{~mL}$ lysate), the soluble fraction was loaded onto a $5 \mathrm{~mL}$ HiTrap SP Sepharose FF column (GE Healthcare) (50 $\mathrm{mM} \mathrm{NaPO}_{4}$ (pH 6.5), $2 \mathrm{mM}$ EDTA). Protein was eluted from the column using a gradient of $1 \mathrm{M} \mathrm{NaCl}$ and T4L99A eluted at $\sim 300 \mathrm{mM} \mathrm{NaCl}$. Pooled fractions were further purified by size-exclusion chromatography using a Superdex S75 gel filtration column (GE Healthcare) (50 mM NaPO 4 (pH 5.5), $2 \mathrm{mM}$ EDTA and $25 \mathrm{mM} \mathrm{NaCl}$ ). Pooled fractions were buffer exchanged into the final NMR buffer (50 $\mathrm{mM} \mathrm{NaPO}_{4}$ (pH 5.5), $2 \mathrm{mM}$ EDTA, $25 \mathrm{mM} \mathrm{NaCl}$ and $2 \mathrm{mM} \mathrm{NaN}_{3}$ ). The NMR sample contained $1.4 \mathrm{mM}$ protein in $95 \% / 5 \%{ }^{1} \mathrm{H}_{2} \mathrm{O} /{ }^{2} \mathrm{H}_{2} \mathrm{O}$.

The uniformly $\left[{ }^{1} \mathrm{H},{ }^{13} \mathrm{C},{ }^{15} \mathrm{~N}\right]$ labelled sample of T4 lysozyme mutant $\mathrm{C} 54 \mathrm{~T} / \mathrm{C} 97 \mathrm{~A} / \mathrm{L} 99 \mathrm{~A}$ (T4L99A) was prepared according to an identical procedure except that the cultures were grown in $1 \mathrm{~L}$ of M9 media made with ${ }^{1} \mathrm{H}_{2} \mathrm{O}$ and using $3 \mathrm{~g} \mathrm{~L}^{-1}{ }^{13} \mathrm{C}_{6}$-glucose as the sole carbon source. The final NMR buffer was composed of $50 \mathrm{mM} \mathrm{NaPO}_{4}(\mathrm{pH} 5.5), 2 \mathrm{mM}$ EDTA, $25 \mathrm{mM}$ $\mathrm{NaCl}, 2 \mathrm{mM} \mathrm{NaN}_{3}$ and $1 \%{ }^{2} \mathrm{H}_{2} \mathrm{O}$, with a protein concentration of $1.1 \mathrm{mM}$.

Isotopically labelled human $\alpha$-synuclein $(\alpha \mathrm{S})$ was expressed and purified as follows. The gene for $\alpha \mathrm{S}$ in the ampicillin-resistant pT7-7 vector, kindly provided by Professor Philipp Selenko at the Weizmann Institute of Science, was transformed into BL21 (DE3) chemically competent E. coli cells using standard techniques. A single colony was used to inoculate a $3 \mathrm{~mL} \mathrm{LB}$ culture, which was grown for $6 \mathrm{~h}$ at $37^{\circ} \mathrm{C}$. This was used to inoculate $100 \mathrm{~mL}$ of minimal M9 media supplemented with $1 \mathrm{~g} \mathrm{~L}^{-1}\left[{ }^{15} \mathrm{~N}\right]$-ammonium chloride and $3 \mathrm{~g} \mathrm{~L}^{-1}\left[{ }^{13} \mathrm{C}_{6}\right]$-glucose as the sole nitrogen and carbon sources, respectively. This culture was grown overnight at $37{ }^{\circ} \mathrm{C}$ and used to inoculate a final $1 \mathrm{~L}$ M9 culture. The final culture was grown to $\mathrm{OD}_{600} \approx 0.7$ before a 
$3 \mathrm{~h}$ induction with $1 \mathrm{mM}$ IPTG at $37{ }^{\circ} \mathrm{C}$. The cells were harvested by centrifugation at $4,000 \mathrm{rpm}$ and $4{ }^{\circ} \mathrm{C}$ for $20 \mathrm{~min}$. Cells were resuspended in $15 \mathrm{~mL}$ lysis buffer $(20 \mathrm{mM}$ Tris, 2 mM EDTA, pH 7 and 1 cOmplete ${ }^{\text {TM }}$ Mini Protease Inhibitor Cocktail tablet) and heated to $90{ }^{\circ} \mathrm{C}$ for $10 \mathrm{~min}$. After pelleting of the cell debris $(20,000 \mathrm{xg}, 20 \mathrm{~min})$ the soluble fraction was loaded onto a 5 mL HiTrap Q FF column (GE Healthcare) (20 mM Tris, 2 mM EDTA, pH 7). $\alpha \mathrm{S}$ was eluted from the column using a gradient of $1 \mathrm{M} \mathrm{NaCl}$ at $\sim 250 \mathrm{mM} \mathrm{NaCl}$. Pooled fractions were buffer exchanged into $\mathrm{H}_{2} \mathrm{O}$ and stored at $-80{ }^{\circ} \mathrm{C}$. NMR samples were made by first thawing the frozen samples and passing through a $100 \mathrm{kDa}$ filter before buffer exchanging into NMR buffer (20 $\mathrm{mM} \mathrm{NaPO}_{4}, 10 \mathrm{mM} \mathrm{NaCl}, 1 \mathrm{mM}$ EDTA, pH 7) using a $10 \mathrm{kDa}$ MWCO concentrator. The NMR samples were passed through a $30 \mathrm{kDa}$ filter to remove any aggregated protein. The NMR sample contained approximately $1.0 \mathrm{mM}$ protein.

For ${ }^{13} \mathrm{C}_{\alpha}$-detected experiments, isotopically labelled human ubiquitin was expressed and purified as described previously ${ }^{[2]}$. Briefly, wild-type human ubiquitin was expressed as untagged protein using pET15 and E. Coli strain BL21 (DE3) in M9 minimal medium containing $\left[{ }^{15} \mathrm{~N}\right]$-ammonium chloride and $\left[{ }^{13} \mathrm{C}_{6}\right]$-glucose as the sole nitrogen and carbon sources to ensure uniform ${ }^{15} \mathrm{~N} /{ }^{13} \mathrm{C}$ labelling. The protein was thein purified by anion exchange using a Q Sepharose resin followed by gel filtration on a Sehpadex G-100 column (GE Healthcare). The final NMR sample was prepared by dialysis into a $95 \%{ }^{1} \mathrm{H}_{2} \mathrm{O}^{-2} \mathrm{H}_{2} \mathrm{O}$ buffer (100 mM KCl, $2 \mathrm{mM}$ Tris, $2 \mathrm{mM}$ formate, $2 \mathrm{mM}$ piperazine, $2 \mathrm{mM}$ imidazole, $0.2 \mathrm{mM} \mathrm{DSS}$ ) at $\mathrm{pH} 8.6$ with a protein concentration of $0.5 \mathrm{mM}$.

For CON experiments, a $1.0 \mathrm{mM}$ NMR sample of $\mathrm{U}-\left[{ }^{13} \mathrm{C},{ }^{15} \mathrm{~N}\right]$-human ubiquitin prepared in 50 $\mathrm{mM} \mathrm{NaPO}$, $\mathrm{pH} 7.0,10 \%{ }^{2} \mathrm{H}_{2} \mathrm{O}$ was purchased from CortecNet, France and used without further purification. 


\section{NMR Acquisition Parameters}

Table S1 Summary of NMR acquisition parameters used for ${ }^{13} \mathrm{C}_{\mathrm{t}^{-}}{ }^{13} \mathrm{C}_{\mathrm{p}}$ correlation experiments and CON experiments.

\begin{tabular}{|l|l|l|l|l|l|l|l|}
\hline${ }^{13} \mathrm{C}_{\mathrm{t}^{13}} \mathrm{C}_{\mathrm{p}}$ correlation experiments carried out on $1.4 \mathrm{mM}\left[{ }^{2} \mathrm{H},{ }^{13} \mathrm{C},{ }^{15} \mathrm{~N}\right]$-labelled T499A \\
\hline $\begin{array}{l}\text { Temp } \\
(\mathrm{K})\end{array}$ & Residue ${ }^{\mathrm{a}}$ & $\begin{array}{l}\mathrm{B}_{0} \text { Field } \\
(\mathrm{T})\end{array}$ & $\begin{array}{l}{ }^{13} \mathrm{C} \mathrm{SW}(\mathrm{ppm}), t_{2} \\
\text { complex points }\end{array}$ & $\begin{array}{l}{ }^{13} \mathrm{C} \mathrm{SW}(\mathrm{ppm}), t_{1} \\
\text { complex points }\end{array}$ & $\begin{array}{l}\mathrm{d} 1^{\mathrm{b}} \\
(\mathrm{sec})\end{array}$ & $\mathrm{NS}^{\mathrm{c}}$ & Timed $^{\mathrm{d}}$ (hours) \\
\hline 298 & Valine & 14.1 & $33.3,562$ & $9.47,40$ & 2.5 & 4 & 0.5 \\
\hline 298 & Isoleucine & 14.1 & $33.3,562$ & $9.47,40$ & 2.5 & 4 & 0.5 \\
\hline
\end{tabular}

\begin{tabular}{|l|l|l|l|l|l|l|l|}
\hline \multicolumn{2}{|l|}{ CON Experiments } \\
\hline $\begin{array}{l}\text { Temp } \\
(\mathrm{K})\end{array}$ & Sample $^{\mathrm{e}}$ & $\begin{array}{l}\mathrm{B}_{0} \text { Field } \\
(\mathrm{T})\end{array}$ & $\begin{array}{l}{ }^{13} \mathrm{C} \mathrm{SW}(\mathrm{ppm}), t_{2} \\
\text { complex points }\end{array}$ & $\begin{array}{l}{ }^{15} \mathrm{~N} \mathrm{SW}(\mathrm{ppm}), t_{1} \\
\text { complex points }\end{array}$ & $\begin{array}{l}\mathrm{d} 1^{\mathrm{b}} \\
(\mathrm{sec})\end{array}$ & $\mathrm{NS}^{\mathrm{c}}$ & Time $^{\mathrm{d}}$ (hours) \\
\hline 298 & Ubq & 11.7 & $23.8,512$ & $35.0,128$ & 1.0 & 16 & 1.3 \\
\hline 298 & $\alpha$-syn & 14.1 & $23.2,512$ & $32.9,128$ & 1.5 & 16 & 1.9 \\
\hline
\end{tabular}

\begin{tabular}{|c|c|c|c|c|c|c|c|c|}
\hline \multicolumn{9}{|c|}{$\mathrm{C} \alpha$ Detect Experiments } \\
\hline $\begin{array}{l}\text { Temp } \\
(\mathrm{K})\end{array}$ & Sample $^{f}$ & $\begin{array}{l}\text { Pulse } \\
\text { Sequenceg }\end{array}$ & $\begin{array}{l}\mathrm{B}_{0} \text { Field } \\
(\mathrm{T})\end{array}$ & $\begin{array}{l}{ }^{13} \mathrm{C}_{\alpha} \mathrm{SW}(\mathrm{ppm}), t_{2} \\
\text { complex points }\end{array}$ & $\begin{array}{l}{ }^{13} \mathrm{CO} \mathrm{SW}(\mathrm{ppm}), t_{1} \\
\text { complex points }\end{array}$ & $\begin{array}{l}\mathrm{d} 1^{\mathrm{b}} \\
(\mathrm{sec})\end{array}$ & $\mathrm{NS}^{\mathrm{c}}$ & $\begin{array}{l}\text { Time }^{\mathrm{h}} \\
\text { (hours) }\end{array}$ \\
\hline 298 & Ubq & c coca & 14.1 & $53.1,1024$ & $14.1,128$ & 2.5 & 128 & 24.0 \\
\hline 298 & Ubq & c coca ia & 14.1 & $53.1,1024$ & $14.1,64$ & 2.5 & 128 & 48.0 \\
\hline 298 & T4L & c_coca & 14.1 & $53.1,512$ & $18.1,128$ & 2.5 & 184 & 34.0 \\
\hline
\end{tabular}

a) ${ }^{13} \mathrm{C}_{\mathrm{t}}{ }^{13} \mathrm{C}_{\mathrm{p}}$ correlation experiments can only probe a single residue type at a time

b) Recovery delay

c) Number of transients (scans) per FID

d) Total acquisition time of the experiment (including in-phase and anti-phase planes)

e) Sample experiment is carried out on. Ubq $=1.0 \mathrm{mM} \mathrm{U}-\left[{ }^{13} \mathrm{C},{ }^{15} \mathrm{~N}\right]$-labelled human ubiquitin, $\alpha$-syn $=$ $1.0 \mathrm{mM} \mathrm{U}-\left[{ }^{13} \mathrm{C},{ }^{15} \mathrm{~N}\right]$-labelled human $\alpha$-Synuclein

f) $\mathrm{Ubq}=0.5 \mathrm{mM} \mathrm{U}-\left[{ }^{13} \mathrm{C},{ }^{15} \mathrm{~N}\right]$-labelled human ubiquitin, T4L $=1.1 \mathrm{mM} \mathrm{U}-\left[{ }^{13} \mathrm{C},{ }^{15} \mathrm{~N}\right]$-labelled T4 lysozyme mutant C54T/C97A/L99A

g) Both c_coca and c_coca_ia pulse sequences are from within the Bruker standard library. See below for details.

h) For c_coca only a single plane is collected wherein the directly acquired ${ }^{13} \mathrm{C}_{\alpha}$ magnetisation is antiphase with respect to the ${ }^{13} \mathrm{CO}$ coupling and in-phase with respect to the ${ }^{13} \mathrm{C}_{\beta}$ coupling. For the c_coca_ia pulse sequence four planes are recorded, giving all in-phase and anti-phase permutations with respect to both couplings. See below for details. 


\section{FID-Net Architecture}

The main principles of the FID-Net DNN architecture have been described previously. The only difference between the architecture of the previous FID-Nets and ones presented here is that the input size has been increased from $512 \times 4$ to $1024 \times 4$. This means that the dimension in the time domain to be decoupled can contain up to 512 complex points. The increased size of the network reflects the increased number of points usually recorded in the direct dimension compared to indirect dimensions. The full architecture used for models here is shown in Fig S1.

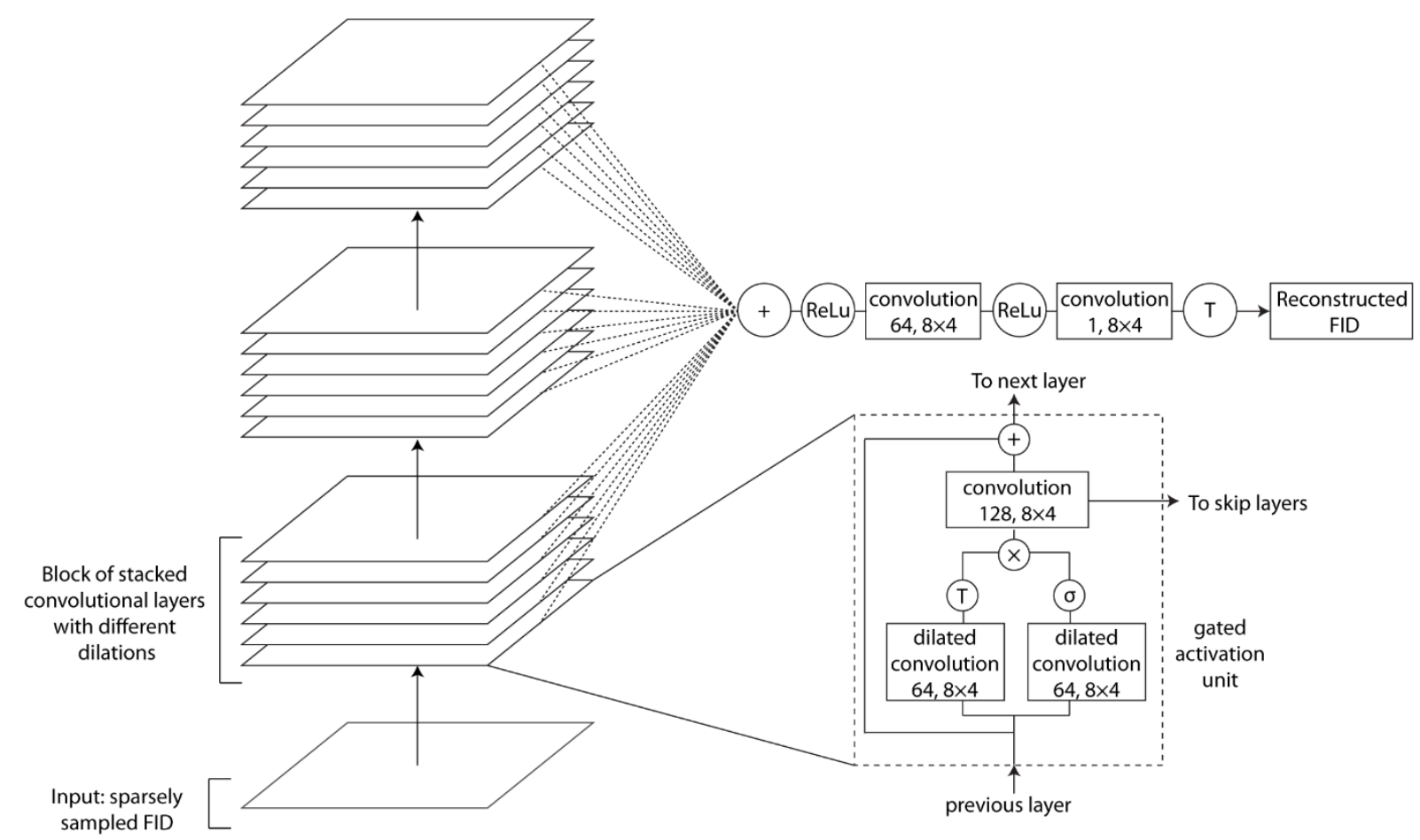

Fig S1 The deep neural network architecture employed within this study, Figure adapted from Karunanithy et al. ${ }^{[3]}$ and further network details are discussed in detail in this paper.

All decoupling networks employ the same architecture but are trained on different data (see below). The architecture is composed of a series of stacked residual units. Each residual unit consists firstly of a dilated convolutional layer composed of 128 filters an $8 \times 4$ kernel size. Half of the filters are activated by a sigmoidal activation function and the other half by a hyperbolic tangent function. The results of these two activations are then multiplied (creating a gated activation unit) and passed through a second convolutional layer, with no dilation, which is also composed of 128 filters and an $8 \times 4$ kernel size. The output of this layer is passed to the 
end layers of the network and also added to the input of the layer to create the input for the next gated activation unit in the network.

The dilations rates employed for FID-Net are cycled through the values: 1, 2, 4, 6, 8, $10,12,14,16,20,24,28,32,40,48,56$ and 64 . These dilation rates are cycled through three times to make the full FID-Net, as indicated by the three blocks in Figure S1.

The outputs of all the individual gated activation units are then summed and passed through a further convolutional layer with rectified linear activation. The final output is produced with a final convolutional layer composed of a single filter followed by hyperbolic tangent activation to ensure the values are between -1 and 1. The full networks contain $39,865,153$ parameters. 


\section{Deep Neural Network Training}

Three networks were trained for the study, all employing the FID-Net architecture (above): One network for decoupling CON spectra, one for $\mathrm{C}_{t}-\mathrm{C}_{\mathrm{p}}$ correlation spectra and one for $\mathrm{C}_{\alpha^{-}}$ detected spectra. The networks are trained exclusively on synthetic data. For the $\mathrm{CON}$ and $\mathrm{C}_{\mathrm{t}^{-}}$ $\mathrm{C}_{\mathrm{p}}$ networks the synthetic data is generated using the equation:

$S\left(t_{1}, t_{2}\right)=\sum_{n} A_{n} \exp \left(-i 2 \pi v_{1, n} t_{1}\right) \exp \left(-R_{2}^{(1, n)} t_{1}\right) \cos \left(\pi J_{n} t_{2}\right) \exp \left(-i 2 \pi v_{2, n} t_{2}\right) \exp \left(-R_{2}^{(2, n)} t_{2}\right)$

Where $n$ runs over the number of signals in the plane, $A_{n}$ is the amplitude of signal $n, v_{1, \mathrm{n}}$ and $v_{2, \mathrm{n}}$ are the frequencies of signal $n$ in the direct and indirect dimensions respectively, $R_{2}^{(1, n)}$ and $R_{2}^{(2, n)}$ are the transverse relaxation rates in the direct and indirect dimensions respectively for signal $n$ and $J_{\mathrm{n}}$ is the $J$ coupling constant for signal $n$. The times $t_{1}$ and $t_{2}$ are given by multiplying $1 / \mathrm{SW}$ (for the relevant sweep width) by the series $0,1, \ldots N-1$ where $N$ is the number of complex points in this dimension.

For the CON network, 500,000 test planes were created for training and 50,000 for cross validation. Each plane contains between 10 and 200 signals at random frequencies with varying relaxation properties (between 5 and $80 \mathrm{~s}^{-1}$ ) and amplitudes. The number of complex points and sweep width in both dimensions are also varied to give a diverse training pool. Each resonance in a plane has a $\mathrm{J}$ coupling between $48-56 \mathrm{~Hz}$. During training $2.5 \%$ of $\mathrm{J}$ couplings are on average set to $0 \mathrm{~Hz}$ to increase robustness of the network.

For the ${ }^{13} \mathrm{C}_{\mathrm{t}^{-1}}{ }^{13} \mathrm{C}_{\mathrm{p}}$ correlation spectra, 250,000 test planes were created for training and 25000 for cross validation. Each plane contains between 10 and 100 signals at random frequencies with varying relaxation properties (between 5 and $80 \mathrm{~s}^{-1}$ ) and amplitudes. The number of complex points and sweep width in both dimensions are also varied to give a diverse training pool. Each resonance in a plane has a J coupling between $28-40 \mathrm{~Hz}$. During training $2.5 \%$ of J couplings are on average set to $0 \mathrm{~Hz}$ to increase robustness of the network.

Full training parameters are given in Table S2 and S3.

The DL models are developed and trained using the Tensorflow library ${ }^{[4]}$ with the Keras frontend $^{[5]}$. For both networks, the training data are phased and Fourier transformed in the indirect dimension and then phased in the direct dimension. Both the real and imaginary parts of the 
spectrum in the direct dimension are retained and are interleaved. As the network decouples four FIDs simultaneously, four consecutive points in the indirect dimension are randomly chosen from each plane as input to the network. This sub-plane is then normalised and random normal noise with standard deviation between 0.001 and 0.15 is added to the input. The inputs are then zero-filled up to 1024 total points in the direct dimension to ensure constant input size for the network.

The cost function used to train the networks is the mean squared error in the frequency domain between the decoupled spectrum produced by the network and the actual decoupled spectrum (ie with all couplings set to $0 \mathrm{~Hz}$ ) and with no noise. Doing training in this way encourages the network to not only decouple the input spectrum but also de-noise the spectrum. The RMSprop optimiser ${ }^{[6]}$ is used for training. Initially the learning rate was set to $10^{-4}$ until the change in validation loss between epochs plateaus. The learning rate was then reduced to $10^{-5}$ and continues until the validation loss plateaus. Training was carried out using NMRBOX ${ }^{[7]}$, with both networks taking approximately two weeks to train on an Nvidia Tesla T4 GPU. The final RMSD for the FIDs produced by the CON network is 0.0183 and for the $\mathrm{C}_{\mathrm{t}}-\mathrm{C}_{\mathrm{p}}$ network is 0.0331 .

Table S2 Parameter ranges used to train the CON spectra decoupling network.

\begin{tabular}{|l|l|}
\hline Number of signals & $10-200$ \\
\hline Amplitude* & $0-2.0$ \\
\hline Direct dimension complex points & $256-512$ \\
\hline Indirect dimension complex points & $56-256$ \\
\hline Direct dimension SW (Hz) & $2500-6000$ \\
\hline Indirect dimension SW (Hz) & $1200-4000$ \\
\hline $\mathrm{J}(\mathrm{Hz})$ & $48.0-56.0 * *$ \\
\hline$R_{2}^{(1)}\left(\mathrm{s}^{-1}\right)$ & $5.0-80.0$ \\
\hline$R_{2}^{(2)}\left(\mathrm{s}^{-1}\right)$ & $5.0-80.0$ \\
\hline
\end{tabular}

*Normal distribution with mean 1.0 and SD 0.5 that is truncated to between $0.0-2.0$

**During training $2.5 \%$ of $\mathrm{J}$ couplings are on average set to $0 \mathrm{~Hz}$. 
Table S3 Parameter ranges used to train the $\mathrm{C}_{\mathrm{t}}-\mathrm{C}_{\mathrm{p}}$ correlation spectra decoupling network.

\begin{tabular}{|l|l|}
\hline Number of signals & $10-100$ \\
\hline Amplitude* & $0-2.0$ \\
\hline Direct dimension complex points & $256-512$ \\
\hline Indirect dimension complex points & $56-256$ \\
\hline Direct dimension SW (Hz) & $3000-12000$ \\
\hline Indirect dimension SW (Hz) & $1000-4000$ \\
\hline $\mathrm{J}(\mathrm{Hz})$ & $26.0-44.0 * *$ \\
\hline$R_{2}^{(1)}\left(\mathrm{s}^{-1}\right)$ & $5.0-80.0$ \\
\hline$R_{2}^{(2)}\left(\mathrm{s}^{-1}\right)$ & $5.0-80.0$ \\
\hline
\end{tabular}

*Normal distribution with mean 1.0 and SD 0.5 that is truncated to between $0.0-2.0$

**During training $2.5 \%$ of $\mathrm{J}$ couplings are on average set to $0 \mathrm{~Hz}$.

For training the network to decouple ${ }^{13} \mathrm{C}_{\alpha}$-detected spectra, the training data must include an additional coupling constant with sine modulation (to match the data produced by the c_coca pulse sequence below). Therefore, equation (1) is augmented to give:

$S\left(t_{1}, t_{2}\right)=\sum_{n} A_{n} \exp \left(-i 2 \pi v_{1, n} t_{1}\right) \exp \left(-R_{2}^{(1, n)} t_{1}\right) \cos \left(\pi J_{n}^{c \alpha-c \beta} t_{2}\right) \sin \left(\pi J_{n}^{C \alpha-c o} t_{2}\right) \exp \left(-i 2 \pi v_{2, n} t_{2}\right) \exp \left(-R_{2}^{(2, n)} t_{2}\right)$

Where $J_{n}^{C \alpha-C \beta}$ and $J_{n}^{C \alpha-C O}$ are the $J$ coupling constants for nucleus $\mathrm{n}$ between ${ }^{13} \mathrm{C}_{\alpha}$ and ${ }^{13} \mathrm{C}_{\beta}$ nuclei and between ${ }^{13} \mathrm{C}_{\alpha}$ and ${ }^{13} \mathrm{CO}$, respectively.

For the ${ }^{13} \mathrm{C}_{\alpha}$-detected spectra, 250,000 test planes were created for training and 25000 for cross validation. Each plane contains between 60 and 200 signals at random frequencies with varying relaxation properties (between 5 and $60 \mathrm{~s}^{-1}$ ) and amplitudes. The number of complex points and sweep width in both dimensions are also varied to give a diverse training pool. As described above, each signal is modulated by two $J$ couplings $\left(J^{C \alpha-C \beta}\right.$ and $\left.J^{C \alpha-C O}\right)$ that are in the range 26.0-42.0 Hz and 47.0-60.0 Hz, respectively. In training, $10 \%$ of $J^{C \alpha-C \beta}$ couplings are randomly set to $0 \mathrm{~Hz}$ to account for glycine residues where this coupling is not present. Full training parameters are provided in Table S4.

The training spectra are processed in an identical to manner to the decoupling spectra except that the noise with standard deviation in the range 0.001-0.1 is added. The cost function used to train the networks is the mean average error in the frequency domain between the decoupled spectrum produced by the network and the actual decoupled spectrum, i.e. with all couplings 
set to $0 \mathrm{~Hz}$, and with no noise. The RMSprop optimiser ${ }^{[6]}$ is used for training. Initially the learning rate was set to $10^{-4}$ until the change in validation loss between epochs plateaus. The learning rate was then reduced to $10^{-5}$ and continues until the validation loss plateaus and then further reduced to $10^{-6}$ until plateauing. Training was carried out on an NVIDIA Geforce RTX 3090 GPU and took approximately one week. The final mean average error for the FIDs produced by the network was 0.0154 .

Table S4 Parameter ranges used to train the ${ }^{13} \mathrm{C}_{\alpha}$-detection decoupling network.

\begin{tabular}{|l|l|}
\hline Number of signals & $60-200$ \\
\hline Amplitude* & $0-2.0$ \\
\hline Direct dimension complex points & $360-512$ \\
\hline Indirect dimension complex points & $64-256$ \\
\hline Direct dimension SW (Hz) & $4000-10000$ \\
\hline Indirect dimension SW (Hz) & $1500-4000$ \\
\hline$J^{C \alpha-C \beta}(\mathrm{Hz})$ & $26.0-42.0 * *$ \\
\hline$J^{C \alpha-C O}(\mathrm{~Hz})$ & $47.0-60.0$ \\
\hline$R_{2}^{(1)}\left(\mathrm{s}^{-1}\right)$ & $5.0-60.0$ \\
\hline$R_{2}^{(2)}\left(\mathrm{s}^{-1}\right)$ & $5.0-60.0$ \\
\hline
\end{tabular}

*Normal distribution with mean 1.0 and SD 0.5 that is truncated to between $0.0-2.0$

**During training $10 \%$ of $J^{C \alpha-C \beta}$ couplings are set to $0 \mathrm{~Hz}$ to account for glycine residues where these couplings are not present. 


\section{Application to experimental spectra}

If using a Bruker spectrometer, the raw FID file (ser) must be converted to nmrPipe format using the DMX flag for FID-Net decoupling to perform correctly.

Prior to input into FID-Net, the direct dimension of the spectrum is phased but the imaginary part is not deleted. The spectrum is then transposed, apodized, zero-filled, phased and Fourier transformed in the indirect dimension. These steps are carried out using NMRPipe ${ }^{[8]}$. As the FID-Net DNN only decouples four FIDs at a time, it is applied as a sliding window across the input to yield the final spectrum. The maximum number of complex points that can be inputted into FID-Net is 512 so if the spectrum contains more complex points than this it is truncated at this point. For spectra containing less than 512 complex points must be zero filled up to this point prior to input into the neural network. The FID-Net output can then be truncated at the actual number of complex points in the spectrum. The FID-Net result can then be apodized, zero-filled and Fourier transformed in the direct dimension to give the final virtually decoupled spectrum. Python scripts for running FID-Net decoupling with the result given in NMRPipe format are available at: https://github.com/gogulan-k/FID-Net and make extensive use of the numpy ${ }^{[9]}$ and nmrGlue ${ }^{[10]}$ packages.

Full IPAP virtual decoupling is carried out using processing scripts with NMRPipe. The ubiquitin DIPAP data shown in Fig 5 is virtually decoupled using the 'splitcomb' macro within Topspin. 


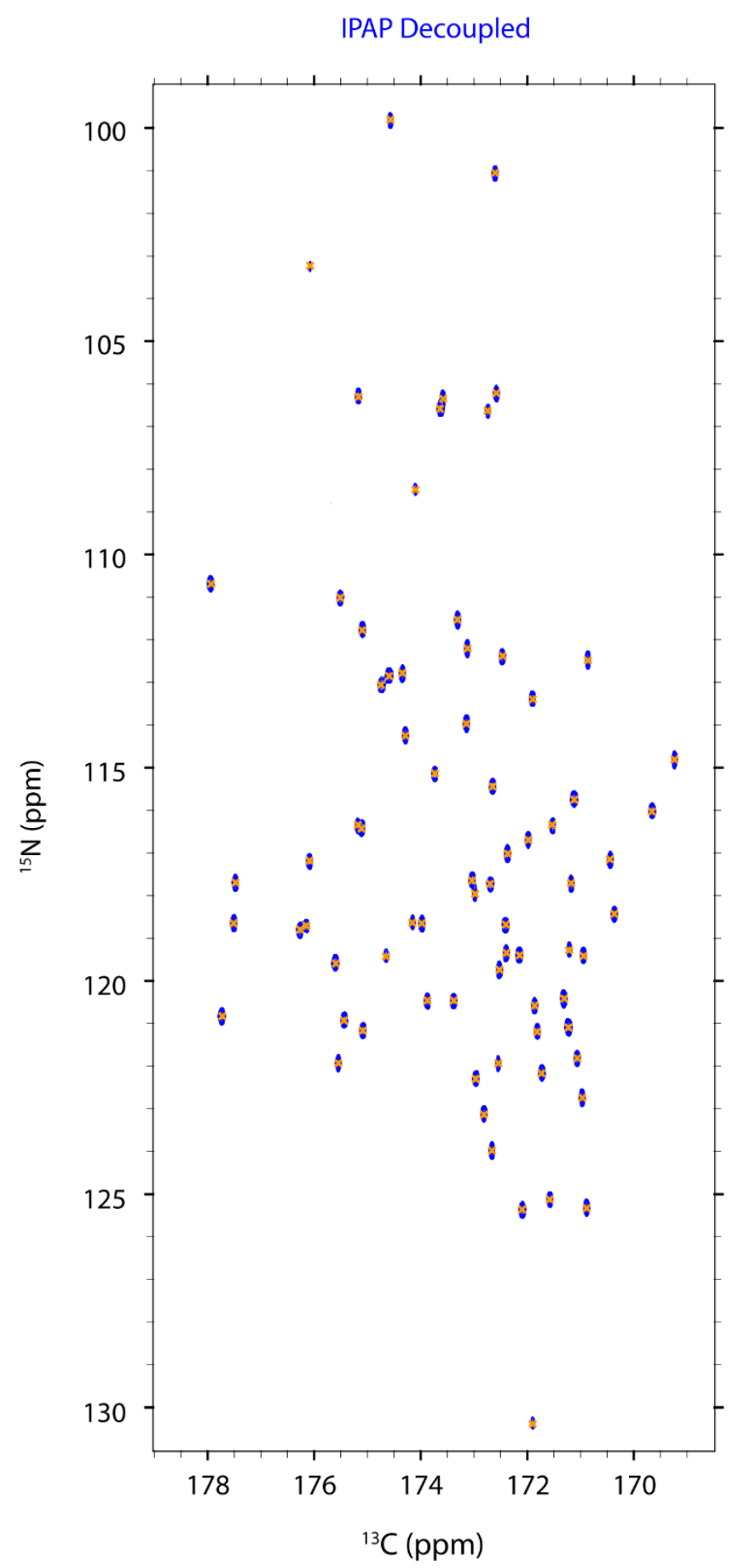

FID-Net Decoupled

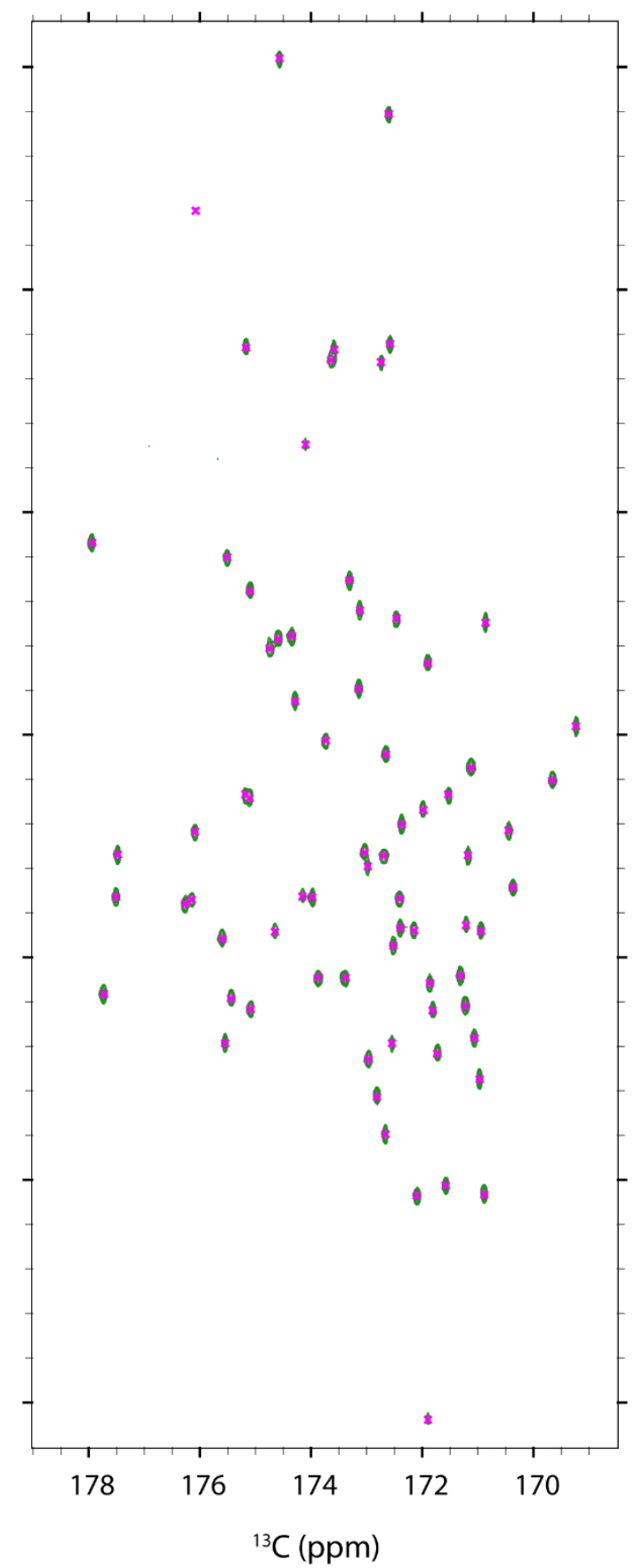

Fig S2 Zoomed in IPAP (blue) and FID-Net (green) decoupled spectra of ubiquitin, with peak positions highlighted in orange and pink, respectively. This demonstrates how all peaks are successfully recovered using the FID-Net decoupling procedure. The figure was generated using NMRFAM-Sparky ${ }^{[13]}$. 

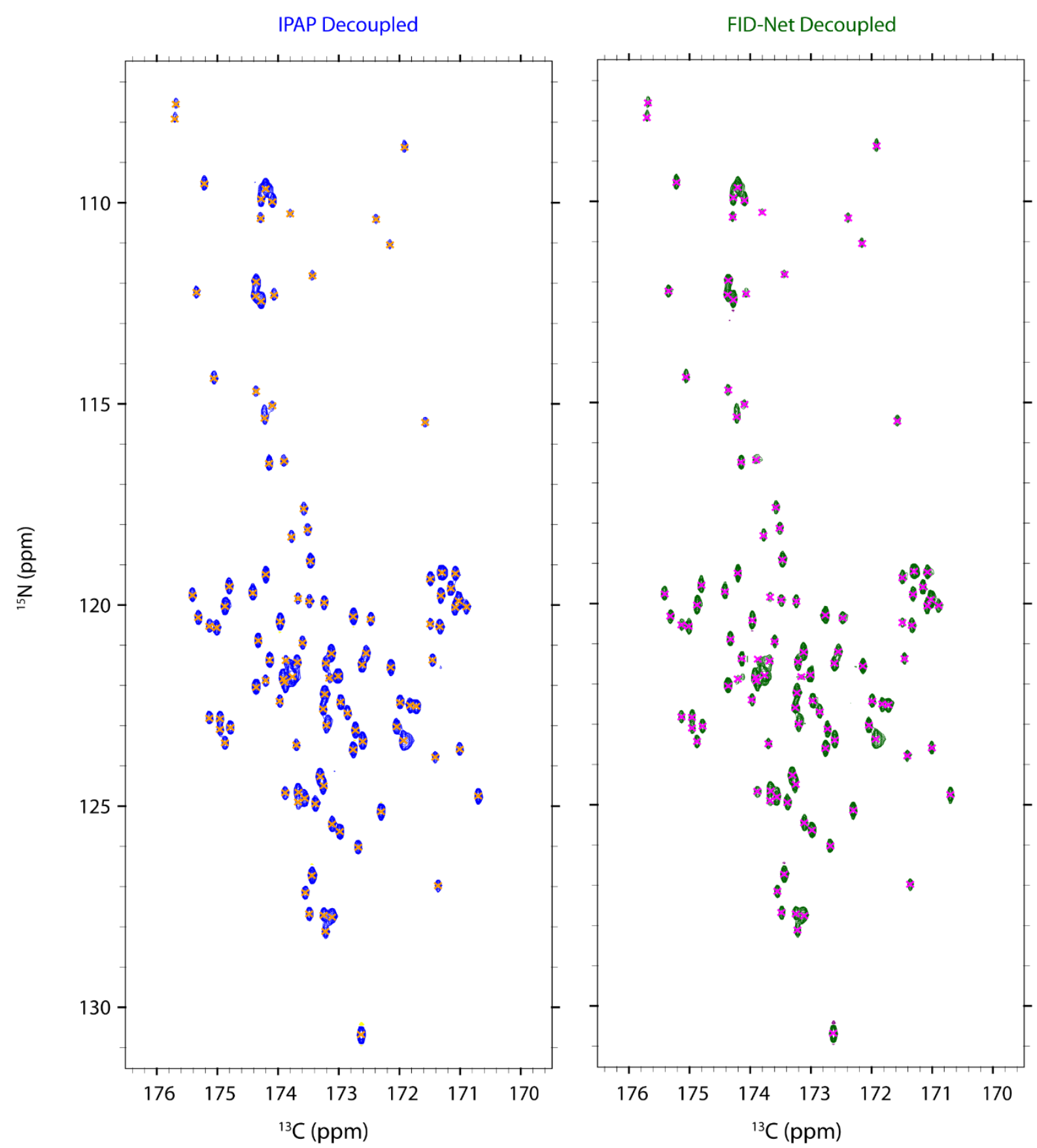

Fig S3 Zoomed in IPAP (blue) and FID-Net (green) decoupled spectra of $\alpha$-synuclein, with peak positions highlighted in orange and pink, respectively. This demonstrates how all peaks are successfully recovered using the FID-Net decoupling procedure. The figure was generated using NMRFAM-Sparky ${ }^{[13]}$. 

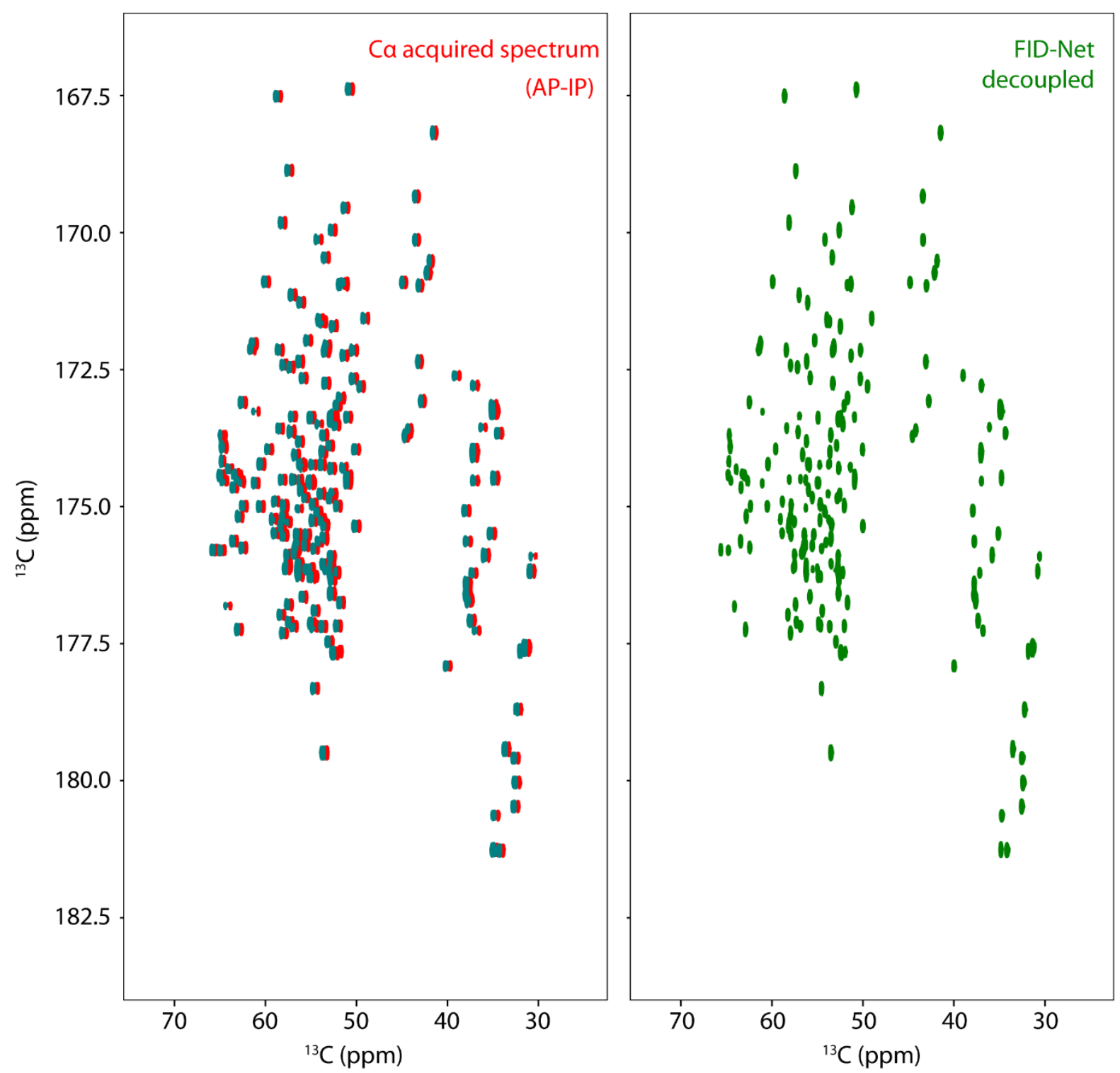

Fig S4 ${ }^{13} \mathrm{C}_{\alpha}$-detected spectrum (red) of $1.1 \mathrm{mM}$ sample of $\left[{ }^{13} \mathrm{C},{ }^{15} \mathrm{~N}\right]$-labelled T4 Lysozyme at 298 K. Positive and negative contours are indicated by the red and teal colours respectively. The spectrum is acquired using the c coca spectrum from the Bruker standard library and the resulting signals are anti-phase with respect to the $J^{C \alpha-C O}$ coupling and in-phase with respect to the $J^{C \alpha-C \beta}$ coupling. The green spectrum shows the successful decoupling of this spectrum using the deep neural network for ${ }^{13} \mathrm{C}_{\alpha}$ acquired spectra. 


\section{Comparison of Pulse Sequences for Recording ${ }^{13} \mathbf{C}_{\alpha}$-detected Spectra}

A key benefit of the deep neural network approach to virtual decoupling outlined here is that it potentially facilitates the use of more simple pulse sequences as only a single spectrum is required. This is in contrast to decoupling approaches such as IPAP or DIPAP where multiple spectra are required with different magnetisation components obtained during acquisition, often needing extended pulse sequences to incorporate this requirement. Using shorter pulse sequences minimises the relaxation losses, while the magnetisation is transverse, leading to higher signal to noise at the start of acquisition.

This feature is demonstrated by the two sequences shown below for obtaining $2 \mathrm{D}^{13} \mathrm{C}_{\alpha^{-}}{ }^{13} \mathrm{CO}$ 2D correlation spectra below, both taken from the Bruker standard pulse sequence library. The c_coca (Fig S5A) is much simpler, featuring only one element where the ${ }^{13} \mathrm{CO}$ chemical shift and ${ }^{13} \mathrm{CO}-{ }^{13} \mathrm{C}_{\alpha}$ scalar coupling evolve. The ${ }^{13} \mathrm{C}_{\alpha}$ chemical shift is then directly acquired giving rise to signals that are anti-phase with respect to the ${ }^{13} \mathrm{CO}-{ }^{13} \mathrm{C}_{\alpha}$ scalar coupling and in-phase with respect to the ${ }^{13} \mathrm{C}_{\alpha^{-}}{ }^{13} \mathrm{C}_{\beta}$ scalar coupling (where this exists), hence can be described as APIP (antiphase, in-phase). As shown in Fig 5 and Fig S4, we can train a DNN to successfully decouple this kind of spectrum, giving singlet-resolved data. As the DNN is only sensitive to the size of the coupling and not the chemical shift of the coupling partner the performance of the decoupling often exceeds what is experimentally achievable (see below).

Conversely the c_coca_ia pulse sequence (Fig S5B) contains the same block as the c_coca but then contains an additional element to create the four spectra required for the DIPAP processing (IP-IP, IP-AP, AP-IP, AP-AP). This additional element in the pulse sequence reduces the signal due to increased relaxation losses and quadruples the effective phase cycle of the experiment. The minimum time for this element using a DIPAP scheme is $\sim 14 \mathrm{~ms}$ whereas using a faster $\mathrm{DS}^{3} \mathrm{E}$ element is $\sim 7 \mathrm{~ms}{ }^{[11,12]}$. Moreover, for serine and threonine residues where ${ }^{13} \mathrm{C}_{\alpha}$ and ${ }^{13} \mathrm{C}_{\beta}$ chemical shifts overlap, decoupling is not possible and signals get distorted, as they are for glycine residues where ${ }^{13} \mathrm{C}_{\beta}$ nuclei are not present. Therefore, as demonstrated in Fig 5 and Fig S4, the FID-Net decoupling approach provides a practical alternative to DIPAP virtual decoupling, facilitating the usage of simpler pulse sequences, substantially reducing the phase cycle of experiments, and providing superior decoupling across all residues. 
A C_coca sequence

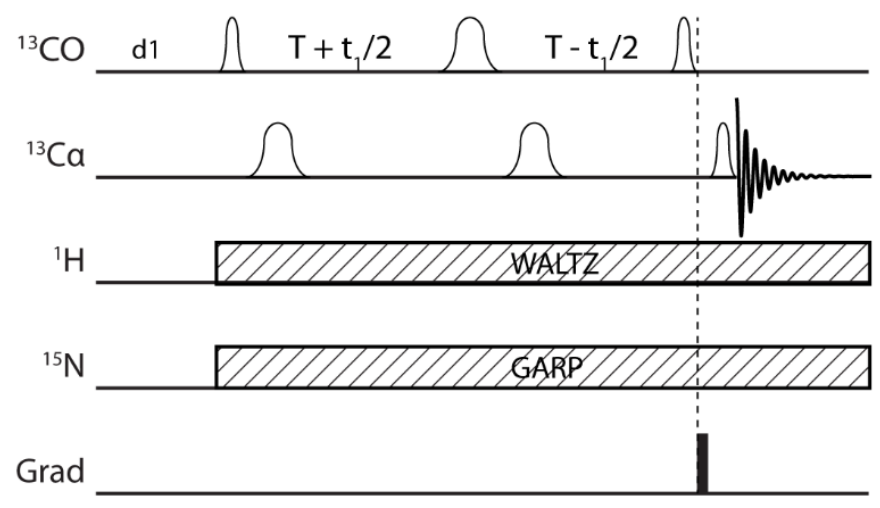

B C_coca_ia sequence

DIPAP block

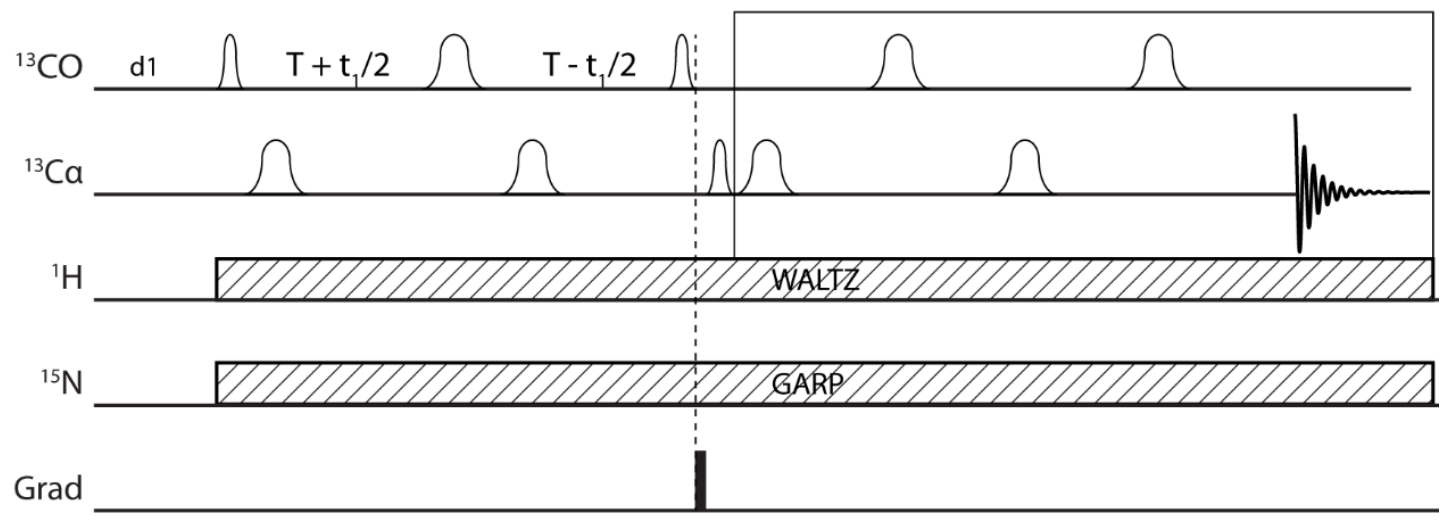

Fig S5 Schematic demonstrating the main differences between the (A) c_coca and (B) c_coca_ia pulse sequences for recording $2 \mathrm{D}^{13} \mathrm{C}_{\alpha}$-detected spectra from the Bruker standard library. Narrow and wide bell shapes indicate band selective $90^{\circ}$ and $180^{\circ}$ pulses respectively. See references for full pulse sequence details ${ }^{[11,12]}$. 


\section{Quantitative Assessment of Virtual Decoupling}

\section{IPAP Decoupling}

Quantitative assessment of the virtual decoupling performance of FID-Net decoupling compared to IPAP is complicated by the non-linear treatment of the data by the neural network. Consequently, we use the NUSCON metrics (https://nuscon.org/metrics.pdf) to assess the spectral information in synthetic spectra. Synthetic in-phase and anti-phase spectra (Fig S6) composed of eight peaks in a row are used in the assessment. The peaks decrease in intensity from 1.0 to 0.075 with increasing linewidth and have $J$-couplings between 50.9 and $54.25 \mathrm{~Hz}$. An additional dataset is also made with identical parameters, but all $J$ couplings set to $0 \mathrm{~Hz}$. The spectrum with no couplings is used to obtain ground truth values for intensity and frequency against which the virtual decoupling and IPAP methods can be evaluated.
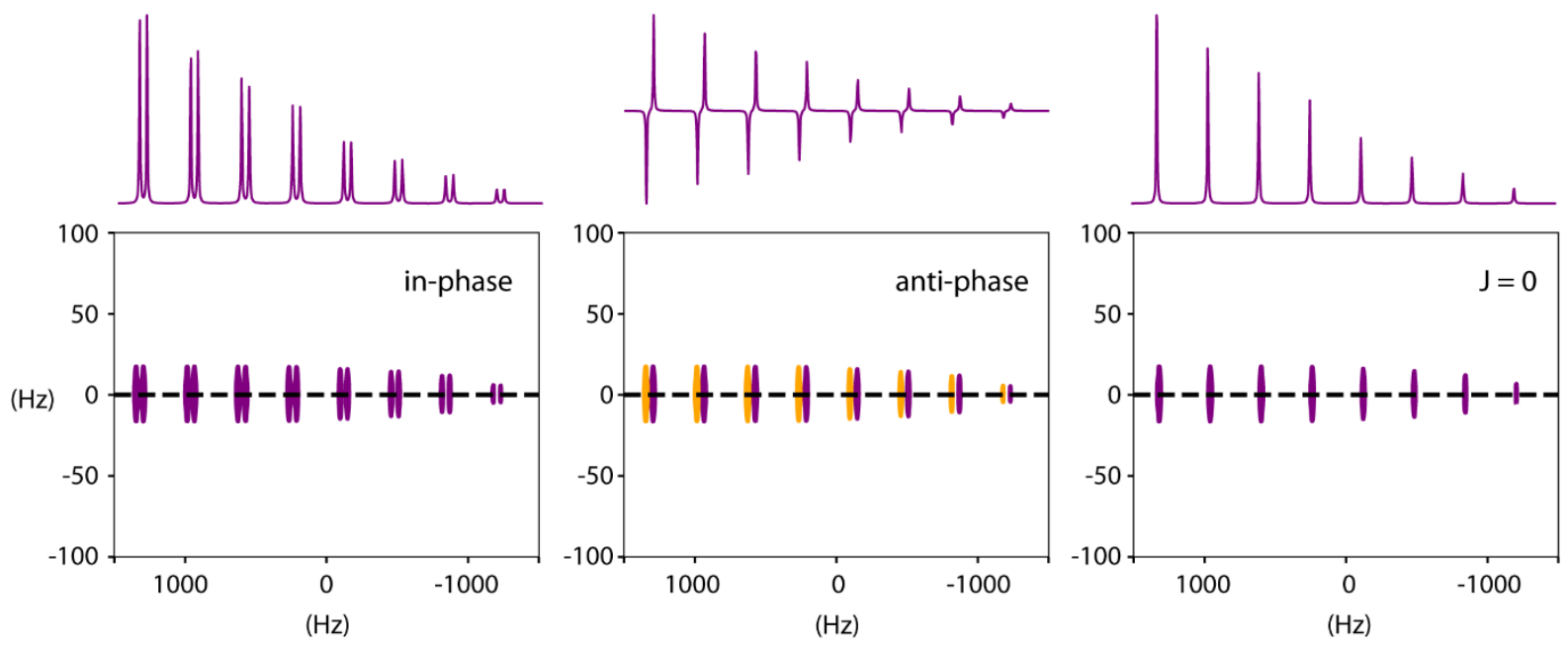

Fig S6 Synthetic spectra used to quantitatively assess the virtual decoupling by FID-Net. The in-phase spectra alone are used as inputs to FID-Net while the in- and anti-phase spectra are processed using the IPAP scheme for comparison. The singlet-resolved spectrum $(J=0 \mathrm{~Hz})$ shown is used to generate a list of ground truth parameters against which the different processing schemes can be compared.

Once the ground truth parameters have been determined, random noise is added to the input in-phase and anti-phase at a given level. The spectra are then processed using either FID-Net (in-phase spectrum only) or IPAP methods. After virtual decoupling, the rescale.com tool within NMRPipe ${ }^{[8]}$ is applied to all transformed spectra so that their maximum intensity is 1.0. The NMRPipe peak picker is used for determining the existence and properties of peaks and these are compared to the reference peaks as described below. This process is repeated one hundred times at each noise level to generate statistics and for each noise level with a given 
processing method the same parameters are used for peak picking. The following NUSCON metrics are used to assess the quality of the decoupling:

1. Linearity of decoupled intensities: Here this is done by measuring the intensity of peaks in the decoupled spectrum and comparing these to do the intensity of peaks in the reference peak list (where $\mathrm{J}=0 \mathrm{~Hz}$ ) by calculating the $R^{2}$, coefficient of determination. The $R^{2}$ calculation assumes there should be a perfectly linear relationship between the intensities of the recovered and master peaks, i.e., we fit to the line $y=x$. Note the values here will in general be different to the square of the correlation coefficient that assumes a model of the form $y=a x+b$ for some numbers $a$ and $b$ with $a \neq 0$. Only where there is a clear correspondence between a reference and recovered peak is this included in the calculation: for each reference peak the corresponding recovered peak (if this exists) is the closest recovered peak within a half-line width distance and all corresponding pairs are mutually exclusive.

2. Accuracy of decoupled frequencies: This is found by calculating the distance between the master and recovered peak positions (in $\mathrm{Hz}$ ) for corresponding pairs of peaks (found as described above). Unlike the NUSCON metric, we only consider pairs where there is a clear correspondence between a master peak and recovered peaks so do not use the symmetric Hausdorff distance (i.e., the correspondence is only considered from master peaks to recovered peaks bringing the metric in line with intensities) and do not need to place an upper bound on the distances since peaks more than a half-linewidth apart would not be considered a pair.

3. False positive rate: this is the rate at which peaks are detected in the decoupled spectrum that are not present in the reference peak list, i.e., the appearance of artefacts.

4. False negative rate: this is the rate at which peaks that appear in the reference peak list are not present in the decoupled spectrum, i.e., missing peaks.

The analysis is carried out at three increasing noise levels shown in Fig S7-S9. Overall, the analysis reveals that FID-Net spectra, decoupled on the basis of the in-phase spectrum alone are very similar in quality to IPAP decoupled spectra (requiring both an in-phase and antiphase spectra) in terms of correctly identifying resonances with the correct intensity and peak positions. As the amount of noise in the underlying spectra increases the ability of the DNN to accurately find peak intensities and positions accurately declines slightly compared to the IPAP decoupled spectra, but the overall spectral information remains very similar. 

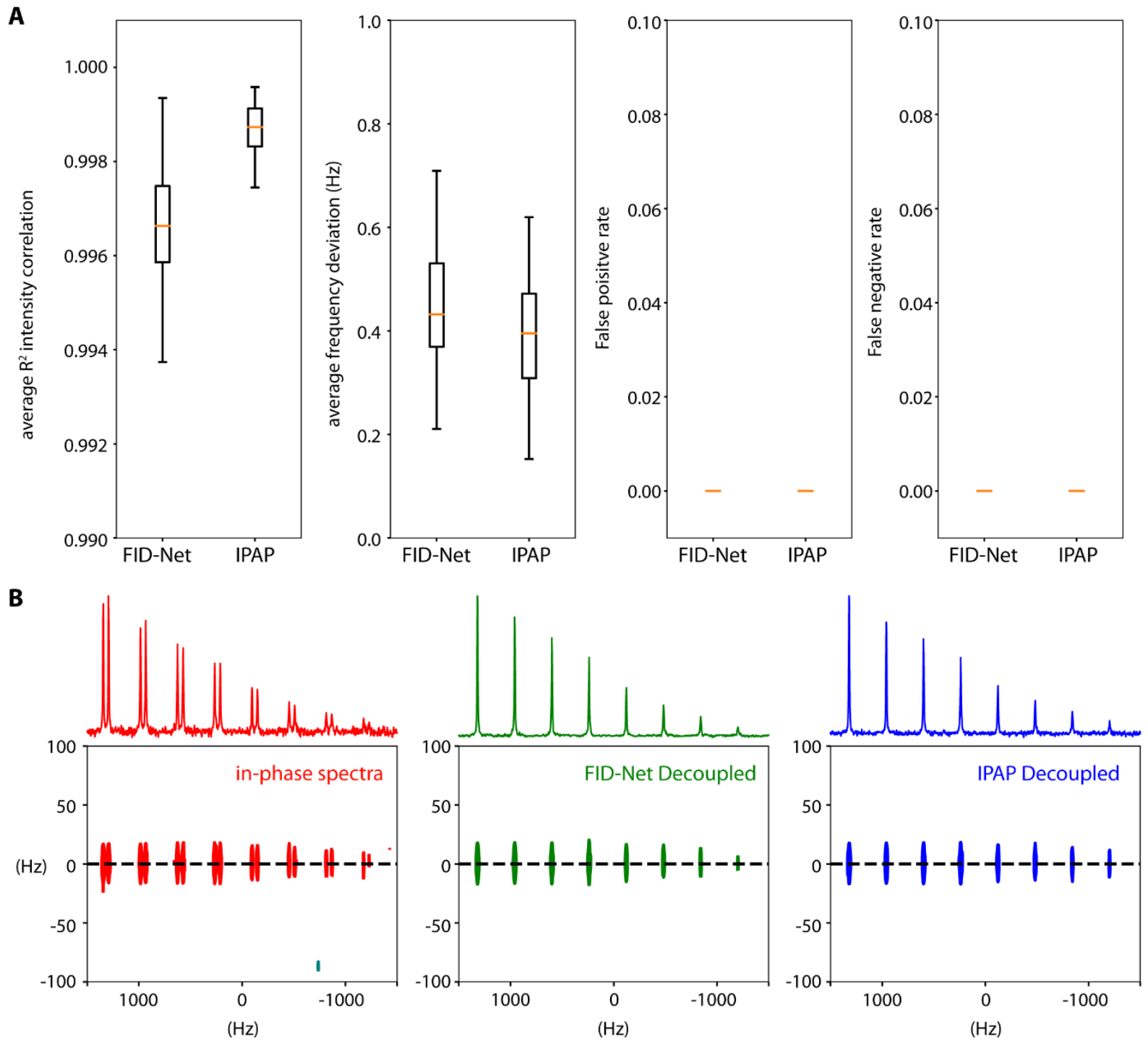

Fig S7 A Box and whisker plots comparing the performance of FID-Net and IPAP virtual decoupling of synthetic data at a noise level of $0.015 \times$ the maximum signal using NUSCON metrics as described above. The results are based on 100 trials where the noise between trials is different but has the same standard deviation. In the box and whisker plots the box indicates the interquartile range, the orange line indicates the median and the top and bottom whiskers extend to the last data point less than third quartile plus $1.5 \times$ inter quartile range and the first data point greater than the first quartile minus $1.5 \times$ inter quartile range. B Exemplar input data and virtually decoupled spectra at this noise level. 
A

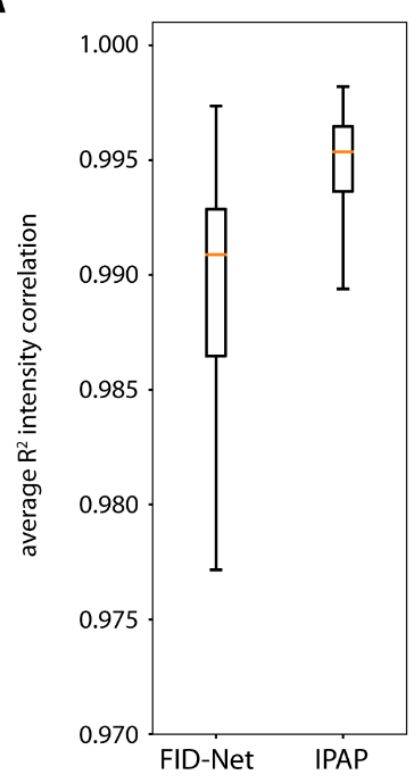

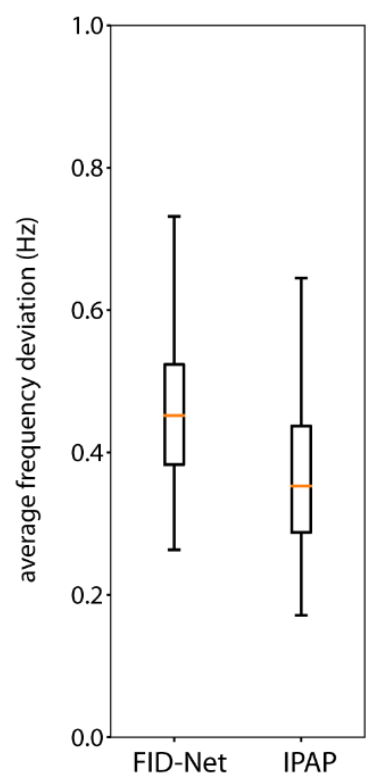
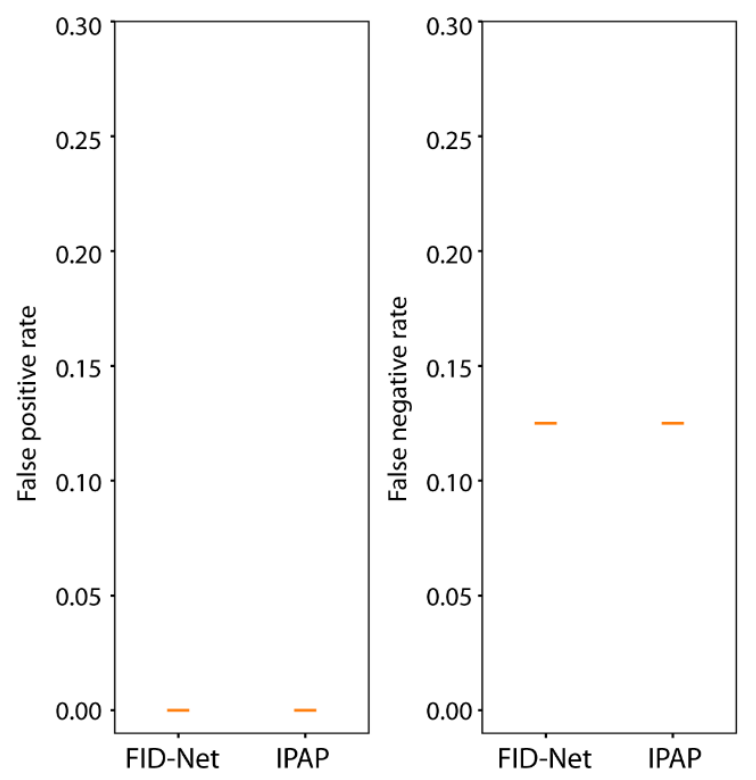

B
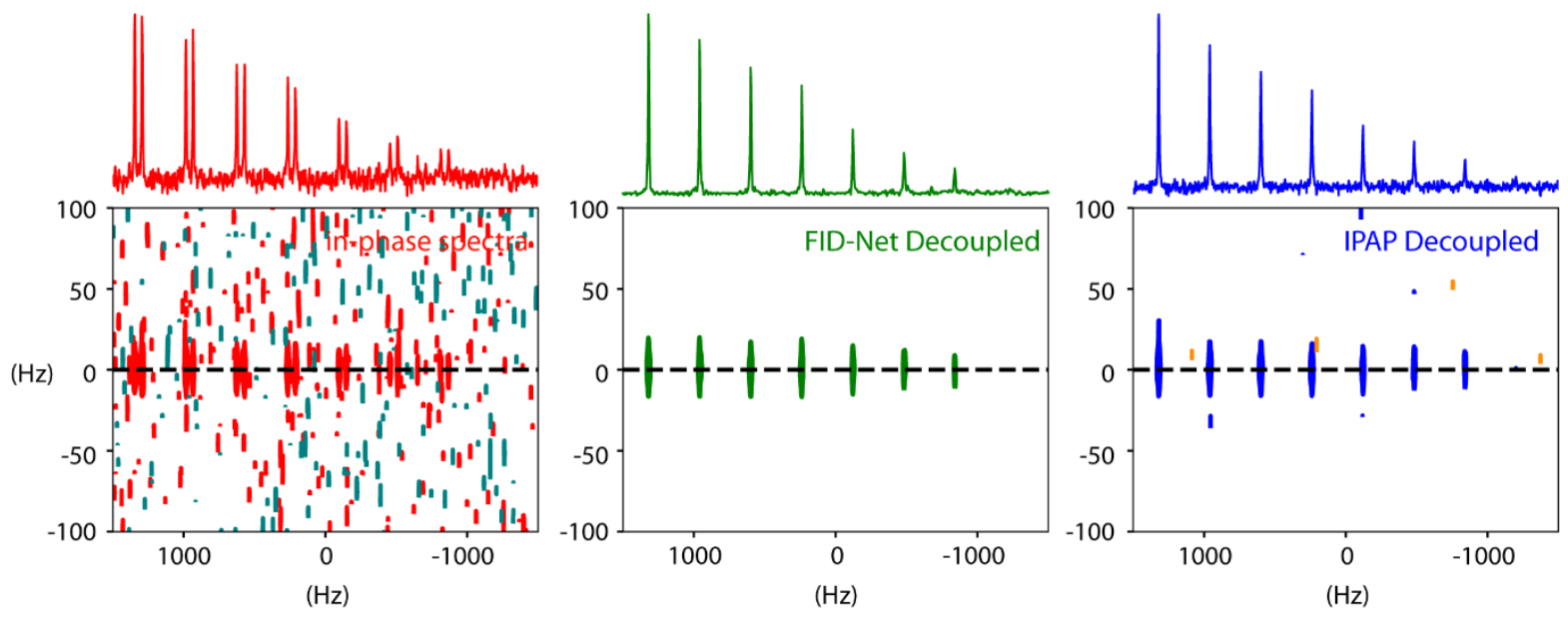

Fig S8 A Box and whisker plots comparing the performance of FID-Net and IPAP virtual decoupling of synthetic data at a noise level of $0.03 \times$ the maximum signal using NUSCON metrics. The box and whisker plots are plotted as described in Fig S7. B Exemplar input data and virtually decoupled spectra at this noise level. 
A

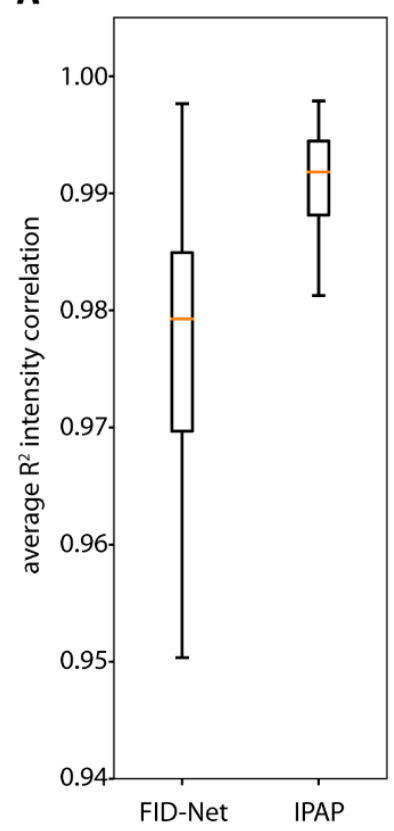

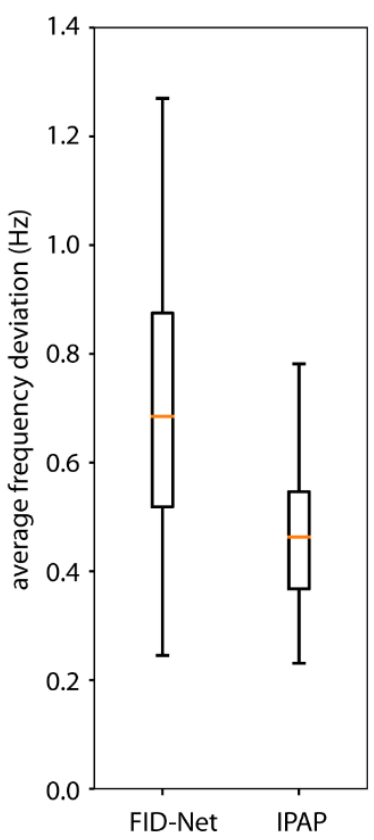
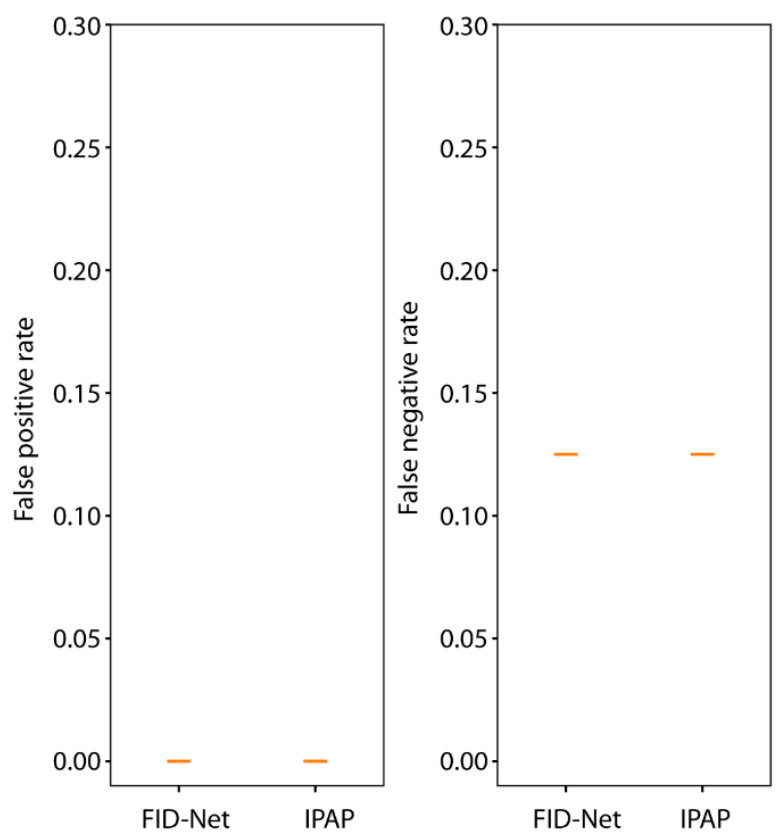

B
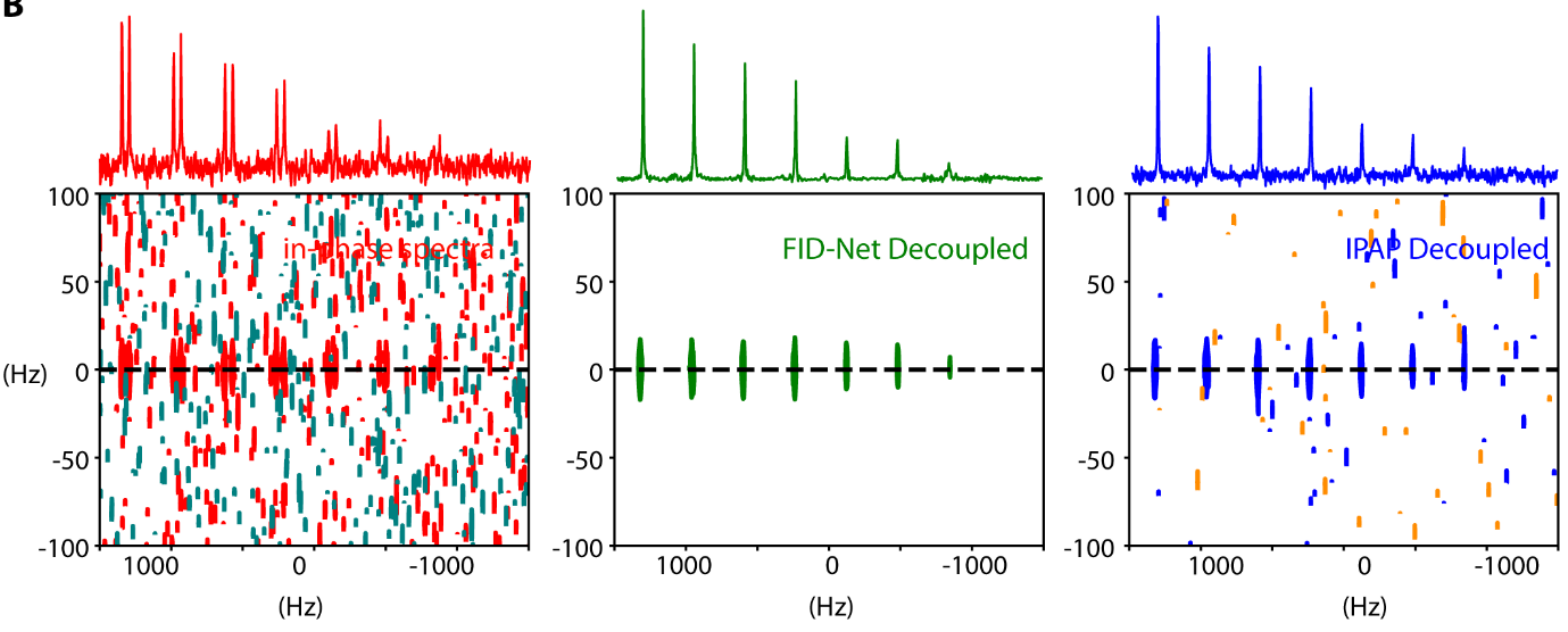

Fig S9 A Box and whisker plots comparing the performance of FID-Net and IPAP virtual decoupling of synthetic data at a noise level of $0.045 \times$ the maximum signal using NUSCON metrics. The box and whisker plots are plotted as described in Fig S7. B Exemplar input data and virtually decoupled spectra at this noise level. 


\section{Decoupling of ${ }^{13} \mathrm{C}_{\alpha^{-}}{ }^{13} \mathrm{CO}$ spectra}

Having performed a quantitative assessment of the neural networks at virtually decoupling a single $J$-coupling during acquisition, below we repeat the analysis for when two couplings can evolve during acquisition. Here, we create synthetic data such as would be obtained in a ${ }^{13} \mathrm{C}_{\alpha^{-}}$ detected experiment using the c_coca pulse sequence as described above. Therefore, the synthetic data is composed of eight signals with declining intensity: six of the signals are antiphase with respect $J^{C \alpha-C O}$ coupling and in-phase with respect to the $J^{C \alpha-C \beta}$ coupling, while the remaining two signals only contain the $J^{C \alpha-C O}$ coupling, emulating glycine signals. The synthetic spectra without added noise is shown in Fig S10 and a corresponding spectra with all couplings set to zero is also shown. This spectrum with $\mathrm{J}=0 \mathrm{~Hz}$ is used to determine a reference peak list against which the virtual decoupling is evaluated and assessed.
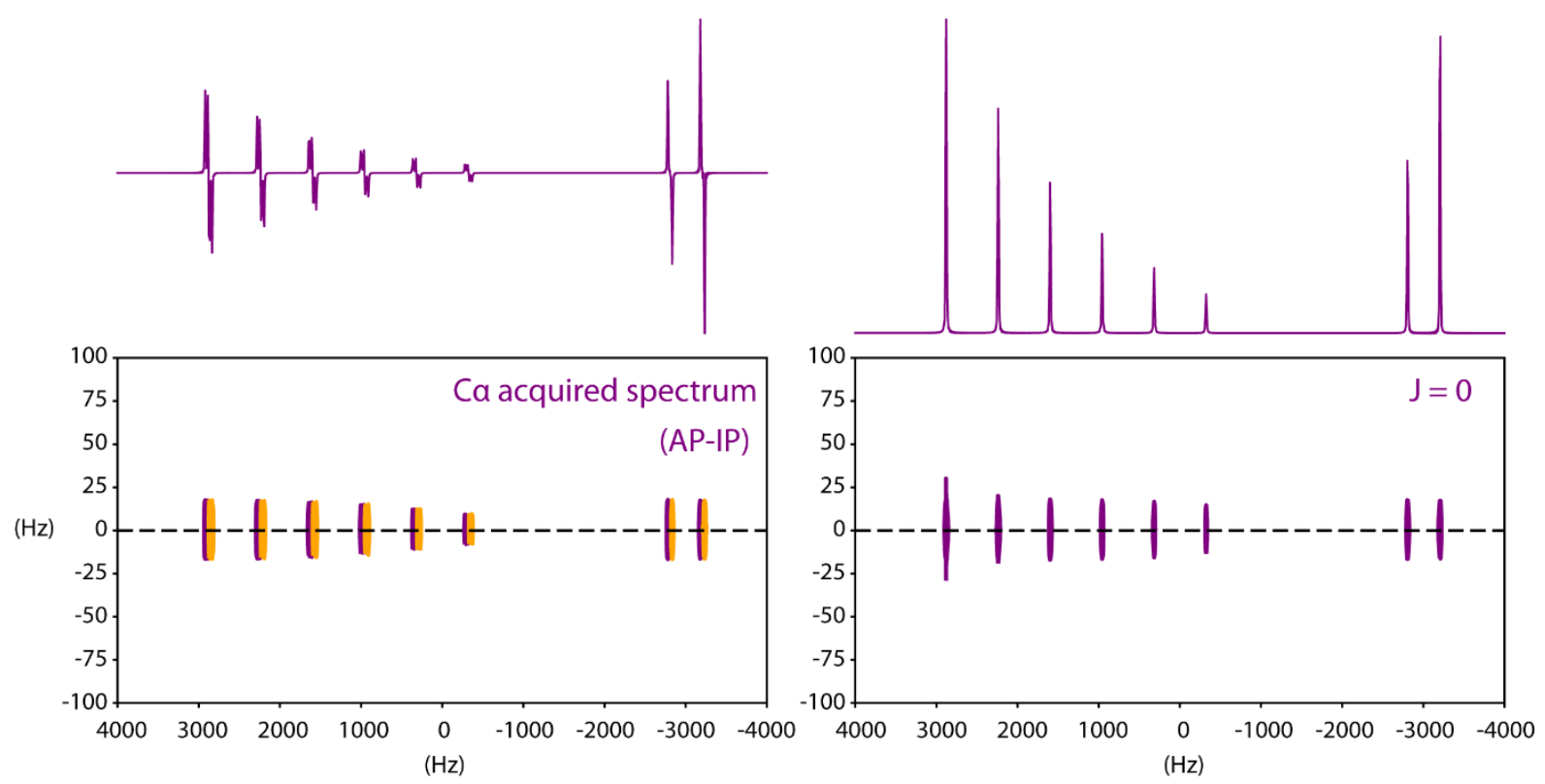

Fig S10 Synthetic spectrum used to test the performance of FID-Net at decoupling ${ }^{13} \mathrm{C}_{\alpha}$ acquired spectra (left), based on the signals obtained using the c_coca pulse sequence. The corresponding spectra with all couplings set to $0 \mathrm{~Hz}$ used to obtain the reference peak list is shown to the right.

Following the same procedure as for the IPAP synthetic data above, the performance of FIDNet virtual decoupling is tested at increasing noise levels using NUSCON metrics. Since the c_coca pulse sequence is incompatible with full DIPAP decoupling and this procedure would not correctly decouple glycine residues a comparison to this is not provided in this case. 
Overall, the results (Fig S11-13) demonstrate that the feasibility of virtually decoupling spectra in the presence of one or two scalar couplings on the basis of a single spectrum. The fidelity of decoupled peak positions is high and where a peak is clearly visible in the input spectrum (above the noise) it is recovered accurately in the decoupled spectrum. Compared to the decoupling of doublet spectra, the linearity of decoupled intensity is lower. The reason for this is that the data for AP-IP data is sine modulated so the intensity of the initial points (about 9 ms) must be extrapolated. On the other hand, for the cosine modulated data in the IPAP examples the intensity of the first points in the FID are unchanged. 

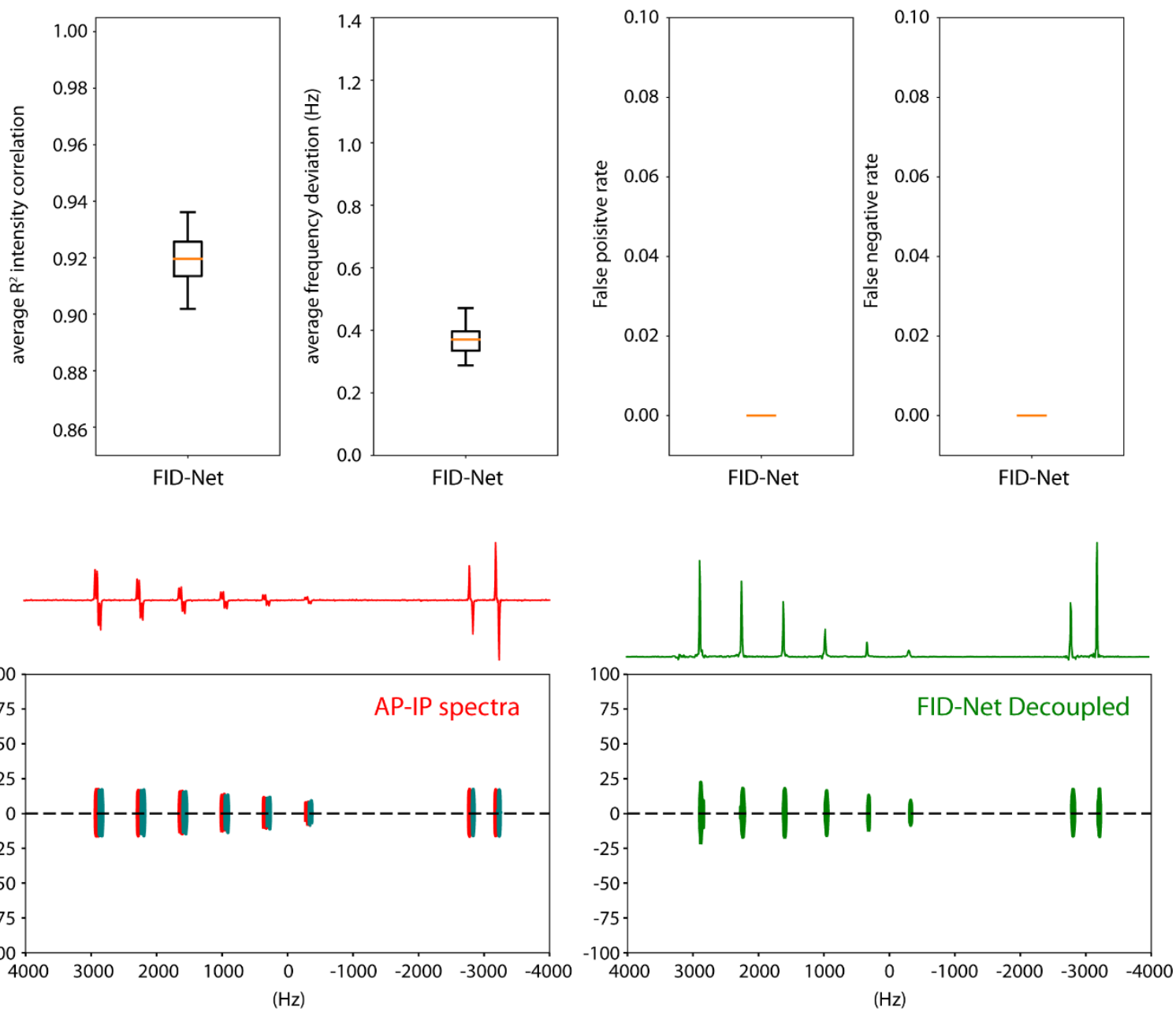

Fig S11 A Box and whisker plots showing the performance of FID-Net virtual decoupling of synthetic data at a noise level of $0.008 \times$ the largest signal using NUSCON metrics. The box and whisker plots are plotted as described in Fig S7. B Exemplar input data and virtually decoupled spectra at this noise level. 

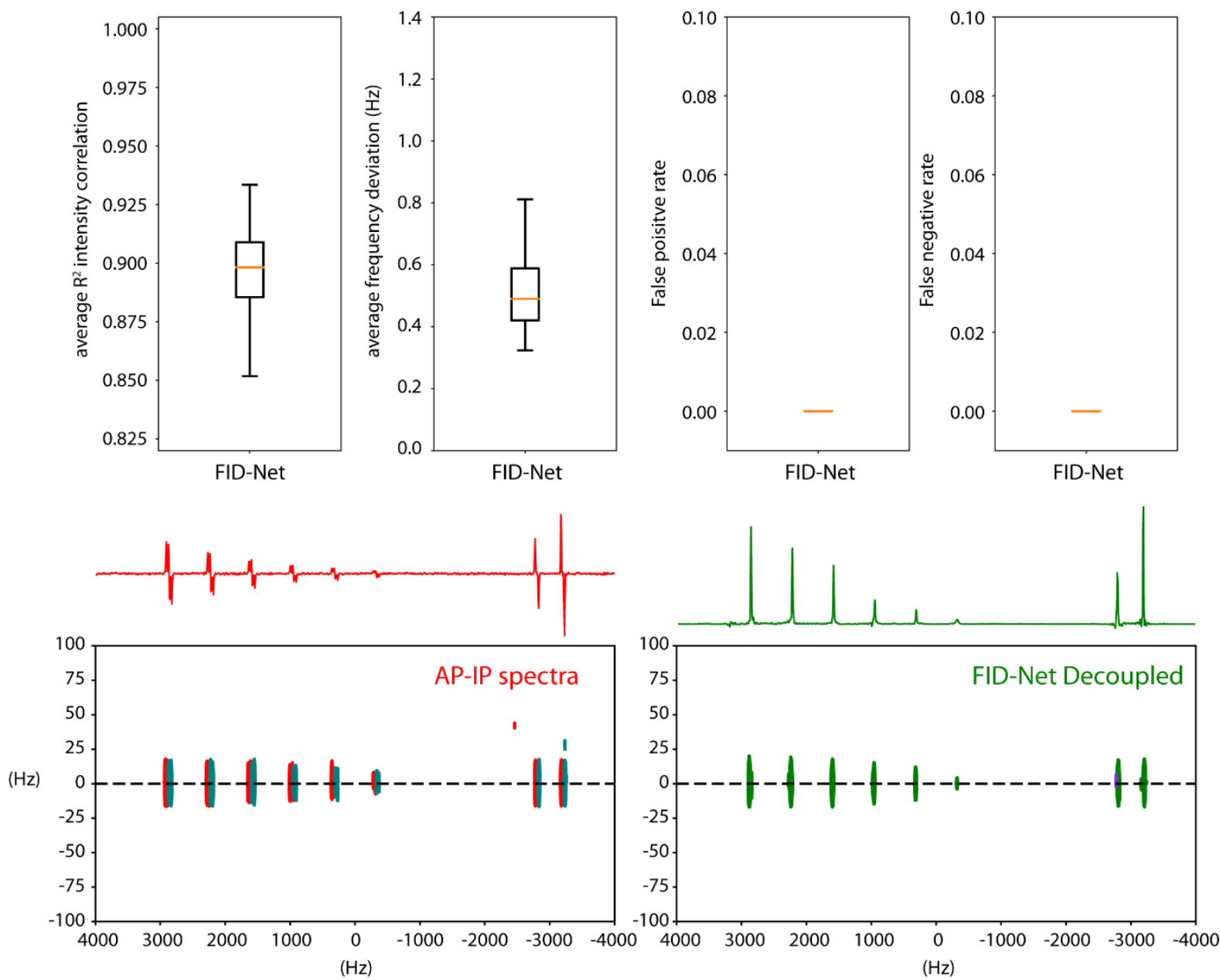

Fig S12 A Box and whisker plots showing the performance of FID-Net virtual decoupling of synthetic data at a noise level of $0.016 \times$ the largest signal using NUSCON metrics. The box and whisker plots are plotted as described in Fig S7. B Exemplar input data and virtually decoupled spectra at this noise level. 

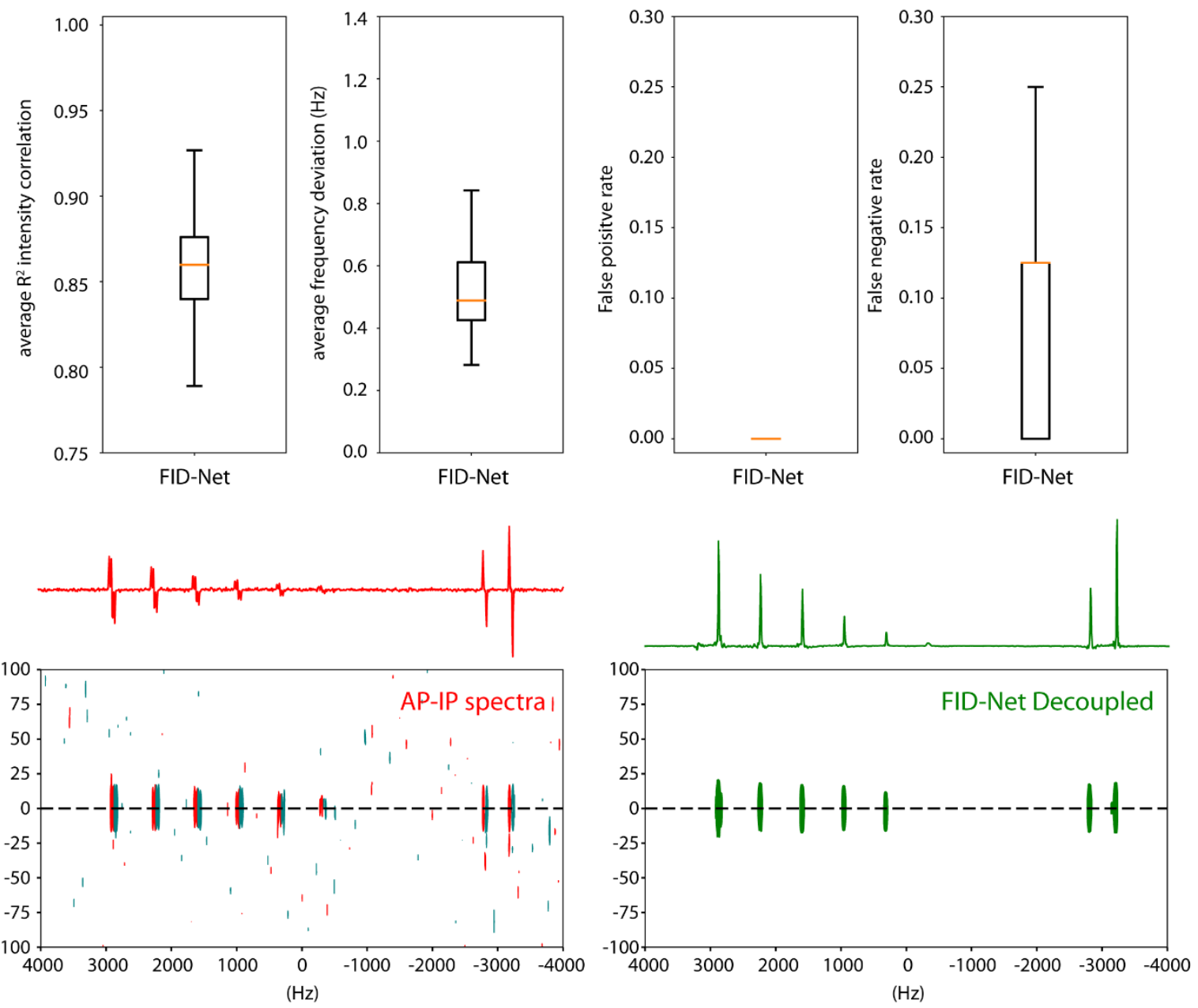

Fig S13 A Box and whisker plots showing the performance of FID-Net virtual decoupling of synthetic data at a noise level of $0.024 \times$ the largest signal using NUSCON metrics. The box and whisker plots are plotted as described in Fig S7. B Exemplar input data and virtually decoupled spectra at this noise level. 


\section{References}

[1] P. Vallurupalli, D. F. Hansen, P. Lundström, L. E. Kay, J. Biomol. NMR 2009, 45, 4555.

[2] A. Oregioni, B. Stieglitz, G. Kelly, K. Rittinger, T. Frenkiel, Sci. Rep. 2017, 7, 1-8.

[3] G. Karunanithy, D. F. Hansen, J. Biomol. NMR 2021, DOI 10.1007/s10858-02100366-w.

[4] M. Abadi, A. Agarwal, P. Barham, E. Brevdo, Z. Chen, C. Citro, G. S. Corrado, A. Davis, J. Dean, M. Devin, et al., 2016.

[5] F. Chollet, others, 2015.

[6] G. Hinton, "Neural Networks for Machine Learning - Lecture 6a - Overview of minibatch Gradient Descent," 2012.

[7] M. W. Maciejewski, A. D. Schuyler, M. R. Gryk, I. I. Moraru, P. R. Romero, E. L. Ulrich, H. R. Eghbalnia, M. Livny, F. Delaglio, J. C. Hoch, Biophys. J. 2017, 112, 1529-1534.

[8] F. Delaglio, S. Grzesiek, G. W. Vuister, G. Zhu, J. Pfeifer, A. Bax, J. Biomol. NMR 1995, 6, 277-293.

[9] C. R. Harris, K. J. Millman, S. J. van der Walt, R. Gommers, P. Virtanen, D. Cournapeau, E. Wieser, J. Taylor, S. Berg, N. J. Smith, et al., Nature 2020, 585, 357362.

[10] J. J. Helmus, C. P. Jaroniec, J. Biomol. NMR 2013, 55, 355-367.

[11] W. Bermel, I. Bertini, L. Duma, I. C. Felli, L. Emsley, R. Pierattelli, P. R. Vasos, Angew. Chemie - Int. Ed. 2005, 44, 3089-3092.

[12] W. Bermel, I. Bertini, I. C. Felli, M. Matzapetakis, R. Pierattelli, E. C. Theil, P. Turano, J. Magn. Reson. 2007, 188, 301-310.

[13] W. Lee, M. Tonelli, J. L. Markley, Bioinformatics 2015, 31, 1325-1327. 